\title{
A uniform analysis of non-symmetric and coercive linear operators
}

\author{
Giancarlo Sangalli \\ Istituto di Matematica Applicata e Tecnologie Informatiche del C.N.R. \\ Via Ferrata 1, 27100 Pavia, Italy \\ sangalli@imati.cnr.it
}

\begin{abstract}
In this work, we show how to construct, by means of the function space interpolation theory, a natural norm $\|\cdot\|$ for a generic linear coercive and non-symmetric operator $\mathcal{L}$. The natural norm $\|\cdot\|||$ allows for continuity and inf-sup conditions which holds independently of $\mathcal{L}$. Particularly we will consider the convection-diffusion-reaction operator, for which we obtain continuity and inf-sup conditions that are uniform with respect to the operator coefficients. In this case, our results give some insight for the analysis of the singular perturbed behavior of the operator, occurring when the diffusivity coefficient is small. Furthermore, our analysis is preliminary to applying some recent numerical methodologies (such as least-squares and adaptive wavelet methods) to this class of operators, and more generally to analyzing any numerical method within the classical framework [1].
\end{abstract}

\section{Introduction}

Consider the convection-diffusion-reaction linear operator

$$
w \mapsto \mathcal{L} w:=-\kappa \Delta w+\beta \cdot \nabla w+\rho w,
$$

where the argument $w$ is a function on the domain $\Omega \subset \mathbb{R}^{n}, \kappa$ is a constant positive diffusion coefficient, $\beta: \Omega \rightarrow \mathbb{R}^{n}$ is a velocity field and $\rho: \Omega \rightarrow \mathbb{R}$ is a reaction coefficient. Under suitable assumptions on the coefficients, e.g., $\rho-1 / 2 \operatorname{div} \beta \geq 0$, the operator $\mathcal{L}$ is an isomorphism from $V:=H_{0}^{1}(\Omega)$ into $V^{*}:=H^{-1}(\Omega)$. In fact, given a source term $f \in V^{*}$, the boundary value problem

$$
\left\{\begin{aligned}
\mathcal{L} u=f & \text { in } \Omega \\
u=0 & \text { on } \partial \Omega
\end{aligned}\right.
$$

admits a unique solution $u \in V$. Nevertheless, the norm of $\mathcal{L}$, as linear operator from $H_{0}^{1}(\Omega)$ into $H^{-1}(\Omega)$,

$$
\|\mathcal{L}\|_{H_{0}^{1}(\Omega) \rightarrow H^{-1}(\Omega)}:=\sup _{w \in H_{0}^{1}(\Omega)} \frac{\|\mathcal{L} w\|_{H^{-1}(\Omega)}}{\|w\|_{H_{0}^{1}(\Omega)}}=\sup _{w \in H_{0}^{1}(\Omega) v \in H_{0}^{1}(\Omega)} \sup _{\|w\|_{H_{0}^{1}(\Omega)}\|v\|_{H_{0}^{1}(\Omega)}},
$$


and the norm of its inverse $\mathcal{L}^{-1}$

$$
\left\|\mathcal{L}^{-1}\right\|_{H^{-1}(\Omega) \rightarrow H_{0}^{1}(\Omega)}:=\sup _{w \in H_{0}^{1}(\Omega)} \frac{\|w\|_{H_{0}^{1}(\Omega)}}{\|\mathcal{L} w\|_{H^{-1}(\Omega)}}=\left(\inf _{w \in H_{0}^{1}(\Omega)} \sup _{v \in H_{0}^{1}(\Omega)} \frac{\langle\mathcal{L} w, v\rangle}{\|w\|_{H_{0}^{1}(\Omega)}\|v\|_{H_{0}^{1}(\Omega)}}\right)^{-1}
$$

depend on the coefficients $\kappa, \beta$ and $\rho$.

Our analysis encompasses any linear and coercive operator $\mathcal{L}$, of which $(1)$ is a model case. Given such an operator $\mathcal{L}$, we construct a norm $\|\cdot\|$ on its domain $V$, such that the continuity

$$
\operatorname{supsup}_{w \in V v \in V} \frac{\langle\mathcal{L} w, v\rangle}{\|w\|\|v\|} \leq \mathcal{C}_{c}<+\infty
$$

and the inf-sup condition

$$
\inf _{w \in V} \sup _{v \in V} \frac{\langle\mathcal{L} w, v\rangle}{\|w\|\|v\|} \geq \mathcal{C}_{i s}>0
$$

hold true with constants $\mathcal{C}_{c}$ and $\mathcal{C}_{\text {is }}$ independent of $\mathcal{L}$. Therefore, for the example (1), $\mathcal{C}_{c}$ and $\mathcal{C}_{\text {is }}$ will be independent of the coefficients $\kappa, \beta$ and $\rho$.

If $\mathcal{L}$ were symmetric, besides coercive, then conditions (3)-(4) would hold true for the so-called energy norm, i.e. by setting $\|w\|:=\langle\mathcal{L} w, w\rangle^{1 / 2}$, with $\mathcal{C}_{c}=\mathcal{C}_{i s}=1$. Our aim is to extend this trivial result to the non-symmetric case, obtaining a suitable $\|\cdot\|$ by means of the function space interpolation.

The norm $\|\cdot\|$, for which (3)-(4) hold true, depends on $\mathcal{L}$ and gives the natural topology for $\mathcal{L}$. For the example (1)-(2), given a source term $f$ and a perturbed source term $f+\delta f$, denoted by $u$ and $u+\delta u$ the solutions of $\mathcal{L} u=f$ and $\mathcal{L}(u+\delta u)=f+\delta f$, respectively, one easily gets from $(3)-(4)$

$$
\frac{\|\delta u\|}{\|u\|} \leq \frac{\mathcal{C}_{c}}{\mathcal{C}_{i s}} \frac{\|\delta f\|_{*}}{\|f\|_{*}}
$$

i.e., the relative perturbation of the solution of (2) is uniformly bounded by the relative perturbation of the source term, $\|\cdot\|_{*}$ being the dual of norm of $\|\cdot\|$. This is the proper framework to understand the behavior of (1)-(2) for small values of the diffusivity $\kappa$, when the higher order term $-\kappa \Delta$ acts as a singular perturbation on the lower order term $\beta \cdot \nabla+\rho$ Id.

Conditions (3)-(4) is also the proper framework for using some recent numerical methodologies for solving (1)-(2). Particularly, we are thinking to the least-squares formulations in the context of finite element methods [6] or in the context of wavelet methods [12], and to adaptive wavelet methods [11] (see also $[2,5,10]$ ).

More generally, (3)-(4) are the starting point for the classical analysis of numerical methods devoted to (1)-(2). When the continuity and inf-sup conditions are known for an operator $\mathcal{L}$, then ideal numerical methods should preserve them at the discrete level. This happens, for example, with symmetric and coercive operators (see [9]) or with some indefinite problems (like in mixed formulations, see [7]), and it is in general the key property for the classical error theory (see, e.g., [1]). Even though there are very effective numerical methods for solving (1)-(2), as the Streamline-Upwind Petrov-Galerkin (SUPG) finite element method (see [8] and [16]), the error 
analysis of them typically do not follow the classical argument mentioned above and it is not completely satisfactory (see [17]). Then we hope this paper could give some insights for a deeper theoretical understanding of numerical methods devoted to (1)-(2) (we refer to [17, §4], [18] and [3, $\S 2.1]$ for a further discussion on the topic).

This work is an extension of our previous analysis proposed in [17], where the convectiondiffusion operator, without the reaction term, is considered. Different estimates for (1)-(2) have been obtained by other authors: see for example the analysis by Bertoluzza, Canuto and Tabacco in $[4, \S 2.1]$, or the paper by Dörfler [13]. The peculiarity of our paper is that both conditions (3)-(4) are obtained for (1)-(2).

The outline of the paper is as follows: in $\S 2$ we present our methodology for obtaining (3)-(4) in the case of a generic non-symmetric and coercive operator $\mathcal{L}$; then we apply the theory first, in $\S 3$, to the very simple one-dimensional $(n=1)$ convection-diffusion-reaction model problem, and then, in $\S 4$, to the multi-dimensional $(n>1)$ case, and discuss the results.

\section{The abstract framework}

In this section, we present our idea for obtaining uniform continuity and inf-sup conditions (3)-(4).

Let $V$ be a Hilbert space, and let $V^{*}$ be its dual. In the present section we consider a generic coercive isomorphism $\mathcal{L}: V \rightarrow V^{*}$ and the associated bilinear form

$$
a(w, v):{ }_{V^{*}}\langle\mathcal{L} w, v\rangle_{V}, \quad \forall w, v \in V ;
$$

The abstract variational problem which correspond to (2) is:

$$
\text { find } u \in V \text { such that } a(u, v)={ }_{V^{*}}\langle f, v\rangle_{V}, \forall v \in V \text {. }
$$

We also assume that $\|\cdot\|_{V}$, the norm of $V$, is the energy norm for $\mathcal{L}$, i.e.

$$
a(w, w)=\|w\|_{V}^{2}, \quad \forall w \in V .
$$

We split $\mathcal{L}=\mathcal{L}_{\text {sym }}+\mathcal{L}_{\text {skew }}$, and introduce the bilinear forms $a_{\text {sym }}(\cdot, \cdot)$ and $a_{\text {skew }}(\cdot, \cdot)$ on $V \times V$ such that

$$
\begin{aligned}
V^{*}\left\langle\mathcal{L}_{\mathrm{sym}} w, v\right\rangle_{V}:=a_{\mathrm{sym}}(w, v):=\frac{1}{2}(a(w, v)+a(v, w)), \quad \forall w, v \in V, \\
{ }_{V^{*}}\left\langle\mathcal{L}_{\text {skew }} w, v\right\rangle_{V}:=a_{\text {skew }}(w, v):=\frac{1}{2}(a(w, v)-a(v, w)), \quad \forall w, v \in V ;
\end{aligned}
$$

in other words $\mathcal{L}_{\text {sym }}$ is the symmetric part of $\mathcal{L}\left(\right.$ i.e., $\left.a_{\text {sym }}(w, v)=a_{\text {sym }}(v, w), \forall w, v \in V\right)$, and we have

$$
\begin{aligned}
a_{\mathrm{sym}}(w, w) & =\|w\|_{V}^{2}, & & \forall w \in V, \\
a_{\mathrm{sym}}(w, v) & \leq\|w\|_{V}\|v\|_{V}, & & \forall w, v \in V,
\end{aligned}
$$

while $\mathcal{L}_{\text {skew }}$ is the skew-symmetric part of $\mathcal{L}$ (i.e., $\left.a_{\text {skew }}(w, v)=-a_{\text {skew }}(v, w), \forall w, v \in V\right)$.

Finally, we define

$$
\begin{aligned}
& \|w\|_{A_{0}}^{2}:=\|w\|_{V}^{2}, \quad \forall w \in V, \\
& \|w\|_{A_{1}}^{2}:=\|w\|_{V}^{2}+\left\|\mathcal{L}_{\text {skew }} w\right\|_{V^{*}}^{2}, \quad \forall w \in V,
\end{aligned}
$$


where

$$
\left\|\mathcal{L}_{\text {skew }} w\right\|_{V^{*}}=\sup _{v \in V} \frac{a_{\text {skew }}(w, v)}{\|v\|_{V}} ;
$$

we also set $A_{0}=A_{1}=V$ from the algebraic standpoint; in other words $A_{0}$ and $A_{1}$ are the same space with the same topology, but the two norms $\|\cdot\|_{A_{0}}$ and $\|\cdot\|_{A_{1}}$ are different (even though equivalent, up to constants depending on $L$ ).

The following lemma states two basic estimates; we explicitly compute the constants appearing into the estimates to put in light their independence of $\mathcal{L}$.

Lemma 1. Under the hypotheses above, we have

$$
\begin{gathered}
a(w, v) \leq 2^{1 / 2}\|w\|_{A_{i}}\|v\|_{A_{1-i}}, \quad \forall w, v \in V, \\
\sup _{v \in V} \frac{a(w, v)}{\|v\|_{A_{1-i}}} \geq 5^{-1 / 2}\|w\|_{A_{i}}, \quad \forall w \in V,
\end{gathered}
$$

for $i=0$ or $i=1$.

Proof. Let $v$ and $w$ be two generic elements of $V$.

By using the Cauchy-Schwartz inequality we easily get

$$
\begin{aligned}
a(w, v) & =a_{\text {sym }}(w, v)+a_{\text {skew }}(w, v) \\
& \leq\|w\|_{V}\|v\|_{V}+\left\|\mathcal{L}_{\text {skew }} w\right\|_{V^{*}}\|v\|_{V} \\
& \leq 2^{1 / 2}\|w\|_{A_{1}}\|v\|_{A_{0}}
\end{aligned}
$$

similarly, since $a_{\text {skew }}(w, v)=-a_{\text {skew }}(v, w)$, we also get $a(w, v) \leq 2^{1 / 2}\|w\|_{A_{0}}\|v\|_{A_{1}}$, then (11) follows.

Recalling (7) and (9), we have

$$
\|w\|_{V} \leq \sup _{v \in V} \frac{a(w, v)}{\|v\|_{V}}
$$

and

$$
\sup _{v \in V} \frac{a_{\text {sym }}(w, v)}{\|v\|_{V}} \leq \sup _{v \in V} \frac{a(w, v)}{\|v\|_{V}} .
$$

Then, we get:

$$
\begin{aligned}
\left\|\mathcal{L}_{\text {skew }} w\right\|_{V^{*}} & =\sup _{v \in V} \frac{a_{\text {skew }}(w, v)}{\|v\|_{V}} \\
& \leq \sup _{v \in V} \frac{a(w, v)}{\|v\|_{V}}+\sup _{v \in V} \frac{a_{\text {sym }}(w, v)}{\|v\|_{V}} \\
& \leq 2 \sup _{v \in V} \frac{a(w, v)}{\|v\|_{V}},
\end{aligned}
$$

and, collecting (13) and (15), we get

$$
\|w\|_{A_{1}} \leq 5^{1 / 2} \sup _{v \in V} \frac{a(w, v)}{\|v\|_{A_{0}}}
$$


which is $(12)$ for $i=1$. We are left to show that

$$
\|w\|_{A_{0}} \leq 5^{1 / 2} \sup _{v \in V} \frac{a(w, v)}{\|v\|_{A_{1}}}
$$

for that purpose, we make use of a duality argument. Reasoning as for (16) we obtain

$$
\|\tilde{w}\|_{A_{1}} \leq 5^{1 / 2} \sup _{v \in V} \frac{a(v, \tilde{w})}{\|v\|_{A_{0}}}
$$

for any $\tilde{w} \in V$. Given a generic $w \in V$, we associate to it $\tilde{w} \in V$ such that $a(v, \tilde{w})=a_{\text {sym }}(v, w)$, $\forall v \in V$; thanks to (18) we have

$$
\|\tilde{w}\|_{A_{1}} \leq 5^{1 / 2} \sup _{v \in V} \frac{a(v, \tilde{w})}{\|v\|_{A_{0}}}=5^{1 / 2} \sup _{v \in V} \frac{a_{\mathrm{sym}}(v, w)}{\|v\|_{A_{0}}}=5^{1 / 2}\|w\|_{A_{0}},
$$

whence

$$
\begin{aligned}
\|w\|_{A_{0}}^{2} & =a_{\mathrm{sym}}(w, w)=a(w, \tilde{w}) \\
& \leq \sup _{v \in V} \frac{a(w, v)}{\|v\|_{A_{1}}} \cdot\|\tilde{w}\|_{A_{1}} \\
& \leq 5^{1 / 2} \sup _{v \in V} \frac{a(w, v)}{\|v\|_{A_{1}}} \cdot\|w\|_{A_{0}},
\end{aligned}
$$

which completes the proof.

From Lemma 1 we can obtain a family of intermediate estimates by means of the function spaces interpolation. We follow the notation and the definitions of [19]; for the reader's convenience, we recall the fundamental definition of interpolated norm, according to the so-called K-method: given $0<\theta<1$ and $1 \leq p \leq+\infty$ we define

$$
\begin{aligned}
& \|w\|_{\left(A_{0}, A_{1}\right)_{\theta, p}}:= \\
& {\left[\int_{0}^{+\infty} \inf _{\substack{w_{0} \in A_{0}, w_{1} \in A_{1}, w_{0}+w_{1}=w}}\left(t^{-\theta}\left\|w_{0}\right\|_{A_{0}}+t^{1-\theta}\left\|w_{1}\right\|_{A_{1}}\right)^{p} \frac{d t}{t}\right]^{\frac{1}{p}} .}
\end{aligned}
$$

Generally $\left(A_{0}, A_{1}\right)_{\theta, p}$ is the space of functions $w \in A_{0}+A_{1}$ such that $\|w\|_{\left(A_{0}, A_{1}\right)_{\theta, p}}<+\infty$. In our particular case, $A_{0}$ and $A_{1}$ are the same space from the algebraic standpoint $\left(A_{0} \equiv A_{1} \equiv V\right)$, and $\|\cdot\|_{\left(A_{0}, A_{1}\right)_{\theta, p}}$ simply is a new norm on $V$.

Lemma 2. Given $\theta, p$ and $p^{\prime}$ such that $0<\theta<1,1 \leq p \leq+\infty$, and $1 / p+1 / p^{\prime}=1$, under the hypotheses above, we have

$$
\begin{gathered}
a(w, v) \leq 2^{1 / 2}\|w\|_{\left(A_{0}, A_{1}\right)_{\theta, p}}\|v\|_{\left(A_{0}, A_{1}\right)_{1-\theta, p^{\prime}}}, \quad \forall w, v \in V, \\
\sup _{v \in V} \frac{a(w, v)}{\|v\|_{\left(A_{0}, A_{1}\right)_{1-\theta, p^{\prime}}}} \geq 5^{-1 / 2}\|w\|_{\left(A_{0}, A_{1}\right)_{\theta, p},}, \quad \forall w \in V .
\end{gathered}
$$


Proof. Typically interpolation theorems are stated in terms of linear operators instead of bilinear forms. Then it is more convenient to rephrase (11) as

$$
\begin{aligned}
& \|\mathcal{L} w\|_{A_{1}^{*}} \leq 2^{1 / 2}\|w\|_{A_{0}}, \\
& \|\mathcal{L} w\|_{A_{0}^{*}} \leq 2^{1 / 2}\|w\|_{A_{1}},
\end{aligned}
$$

and (12) as

$$
\begin{aligned}
\|w\|_{A_{0}} & \leq 5^{1 / 2}\|\mathcal{L} w\|_{A_{1}^{*}} \\
\|w\|_{A_{1}} & \leq 5^{1 / 2}\|\mathcal{L} w\|_{A_{0}^{*}}
\end{aligned}
$$

for all $w \in V$.

From (22) and thanks to Theorem [19, §1.3.3] and [19, §1.11.2], we get (20). Proceeding similarly for $\mathcal{L}^{-1}$, from $(23)$ we obtain

$$
\left\|\mathcal{L}^{-1} \phi\right\|_{\left(A_{0}, A_{1}\right)_{1-\theta, p^{\prime}}^{*}} \leq 5^{1 / 2}\|\phi\|_{\left(A_{0}, A_{1}\right)_{\theta, p}},
$$

for any $\phi \in V^{*}$, that gives $(21)$.

Thanks to $(9), \mathcal{L}_{\text {sym }}$ is an isomorphism from $V$ into $V^{*} \equiv \mathcal{L}_{\text {sym }}(V)$; henceforth, we also assume that $\mathcal{L}_{\text {skew }}$ is injective. Then we introduce the two Hilbert spaces $C_{0}$ and $C_{1}$ :

$$
\begin{aligned}
& C_{0}:=\mathcal{L}_{\text {skew }}(V), \text { with }\|\phi\|_{C_{0}}:=\left\|\mathcal{L}_{\text {skew }}^{-1} \phi\right\|_{V} \\
& C_{1}:=\mathcal{L}_{\text {sym }}(V), \quad \text { with }\|\phi\|_{C_{1}}:=\left\|\mathcal{L}_{\text {sym }}^{-1} \phi\right\|_{V}=\|\phi\|_{V^{*}} .
\end{aligned}
$$

In the next lemma we analyze the structure of $\|\cdot\|_{\left(A_{0}, A_{1}\right)_{\theta, p}}$.

Lemma 3. Given $\theta, p$ and $p^{\prime}$ such that $0<\theta<1,1 \leq p \leq+\infty$, and $1 / p+1 / p^{\prime}=1$, under the hypotheses above, we have

$$
1 / 10\|w\|_{\left(A_{0}, A_{1}\right)_{\theta, p}}^{2} \leq\|w\|_{V}^{2}+\left\|\mathcal{L}_{\text {skew }} w\right\|_{\left(C_{0}, C_{1}\right)_{\theta, p}}^{2} \leq 2\|w\|_{\left(A_{0}, A_{1}\right)_{\theta, p}}^{2}, \quad \forall w \in V .
$$

Proof. Since $\|w\|_{V} \leq\|w\|_{A_{i}}$ with $i=0,1$, then $\|w\|_{V} \leq\|w\|_{\left(A_{0}, A_{1}\right)_{\theta, p}}$ follows by a straightforward application of the interpolation theorem (e.g., [19, §1.3.3]). We also have

$$
\begin{aligned}
\left\|\mathcal{L}_{\text {skew }} w\right\|_{C_{0}} & \leq\|w\|_{A_{0}}, \\
\left\|\mathcal{L}_{\text {skew }} w\right\|_{C_{1}} & \leq\|w\|_{A_{1}},
\end{aligned}
$$

which gives $\left\|\mathcal{L}_{\text {skew }} w\right\|_{\left(C_{0}, C_{1}\right)_{\theta, p}} \leq \mathcal{C}\|w\|_{\left(A_{0}, A_{1}\right)_{\theta, p}}$, whence $\|w\|_{V}^{2}+\left\|\mathcal{L}_{\text {skew }} w\right\|_{\left(C_{0}, C_{1}\right)_{\theta, p}}^{2} \leq 2\|w\|_{\left(A_{0}, A_{1}\right)_{\theta, p}}^{2}$.

In order to complete the proof, we directly deal with the definition of interpolated norm (19). For any $t>0$ consider the two splitting

$$
\begin{aligned}
& w=\tilde{w}_{0}(t)+\tilde{w}_{1}(t), \text { with } \tilde{w}_{i}(t) \in V, i=1,2, \\
& w=\hat{w}_{0}(t)+\hat{w}_{1}(t), \text { with } \hat{w}_{i}(t) \in V, i=1,2 ;
\end{aligned}
$$

then define $w_{0}(t) \in V$ and $w_{1}(t) \in V$ such that $\mathcal{L} w_{i}(t)=\mathcal{L}_{\text {sym }} \tilde{w}_{i}(t)+\mathcal{L}_{\text {skew }} \hat{w}_{i}(t)$, i.e.,

$$
a\left(w_{i}(t), v\right)=a_{\mathrm{sym}}\left(\tilde{w}_{i}(t), v\right)+a_{\text {skew }}\left(\hat{w}_{i}(t), v\right), \quad \forall v \in V, i=0,1,
$$


whence $w=w_{0}(t)+w_{1}(t), \forall t>0$.

Thanks to (12) and to the properties of $a_{\text {sym }}(\cdot, \cdot)$ and $a_{\text {skew }}(\cdot, \cdot)$ we have

$$
\begin{aligned}
\left\|w_{0}(t)\right\|_{A_{0}} & \leq 5^{1 / 2} \sup _{v \in V} \frac{a\left(w_{0}(t), v\right)}{\|v\|_{A_{1}}} \\
& \leq 5^{1 / 2}\left(\sup _{v \in V} \frac{a_{\text {sym }}\left(\tilde{w}_{0}(t), v\right)-a_{\text {skew }}\left(v, \hat{w}_{0}(t)\right)}{\|v\|_{A_{1}}}\right) \\
& \leq 5^{1 / 2}\left(\sup _{v \in V} \frac{a_{\text {sym }}\left(\tilde{w}_{0}(t), v\right)}{\|v\|_{V}}+\sup _{v \in V} \frac{a_{\text {skew }}\left(v, \hat{w}_{0}(t)\right)}{\left\|\mathcal{L}_{\text {skew }} v\right\|_{V^{*}}}\right) \\
& \leq 5^{1 / 2}\left(\left\|\tilde{w}_{0}(t)\right\|_{V}+\left\|\hat{w}_{0}(t)\right\|_{V}\right) .
\end{aligned}
$$

In a similar way, we have

$$
\begin{aligned}
\left\|w_{1}(t)\right\|_{A_{1}} & \leq 5^{1 / 2} \sup _{v \in V} \frac{a\left(w_{1}(t), v\right)}{\|v\|_{A_{0}}} \\
& \leq 5^{1 / 2}\left(\sup _{v \in V} \frac{a_{\text {sym }}\left(\tilde{w}_{1}(t), v\right)+a_{\text {skew }}\left(\hat{w}_{1}(t), v\right)}{\|v\|_{A_{0}}}\right) \\
& \leq 5^{1 / 2}\left(\sup _{v \in V} \frac{a_{\text {sym }}\left(\tilde{w}_{1}(t), v\right)}{\|v\|_{V}}+\sup _{v \in V} \frac{a_{\text {skew }}\left(\hat{w}_{1}(t), v\right)}{\|v\|_{V}}\right) \\
& \leq 5^{1 / 2}\left(\left\|\tilde{w}_{1}(t)\right\|_{V}+\left\|\mathcal{L}_{\text {skew }} \hat{w}_{1}(t)\right\|_{V^{*}}\right) .
\end{aligned}
$$

From (19), by the triangle inequality and using (28)-(29), we have

$$
\begin{aligned}
\|w\|_{\left(A_{0}, A_{1}\right)_{\theta, p} \leq} & {\left[\int_{0}^{+\infty}\left(t^{-\theta}\left\|w_{0}(t)\right\|_{A_{0}}+t^{1-\theta}\left\|w_{1}(t)\right\|_{A_{1}}\right)^{p} \frac{d t}{t}\right]^{1 / p} } \\
\leq & 5^{1 / 2}\left[\int _ { 0 } ^ { + \infty } \left(t^{-\theta}\left\|\tilde{w}_{0}(t)\right\|_{V}+t^{-\theta}\left\|\hat{w}_{0}(t)\right\|_{V}\right.\right. \\
& \left.\left.\quad+t^{1-\theta}\left\|\tilde{w}_{1}(t)\right\|_{V}+t^{1-\theta}\left\|\mathcal{L}_{\text {skew }} \hat{w}_{1}(t)\right\|_{V^{*}}\right)^{p} \frac{d t}{t}\right]^{1 / p} \\
\leq & 5^{1 / 2}\left[\int_{0}^{+\infty}\left(t^{-\theta}\left\|\tilde{w}_{0}(t)\right\|_{V}+t^{1-\theta}\left\|\tilde{w}_{1}(t)\right\|_{V}\right)^{p} \frac{d t}{t}\right]^{1 / p} \\
& +\left[\int_{0}^{+\infty}\left(t^{-\theta}\left\|\mathcal{L}_{\text {skew }} \hat{w}_{0}(t)\right\|_{C_{0}}+t^{1-\theta}\left\|\mathcal{L}_{\text {skew }} \hat{w}_{1}(t)\right\|_{C_{1}}\right)^{p} \frac{d t}{t}\right]^{1 / p} ;
\end{aligned}
$$

finally, taking the infimum over all $\tilde{w}_{0} \in V, \tilde{w}_{1}=w-\tilde{w}_{0} \in V, \hat{w}_{0} \in V$ and $\hat{w}_{1}=w-\hat{w}_{0} \in V$, and using $\left[19,1.3 .3\right.$.(f)], we finally get $\|w\|_{\left(A_{0}, A_{1}\right)_{\theta, p}} \leq 5^{1 / 2}\left(\|w\|_{V}+\left\|\mathcal{L}_{\text {skew }} w\right\|_{\left(C_{0}, C_{1}\right)_{\theta, p}}\right)$, completing the proof of (25).

When $p=p^{\prime}=2$ and $\theta=1-\theta=1 / 2$, Lemma 2 gives the continuity and inf-sup conditions for $\mathcal{L}$, as stated in the introduction, where $\|\cdot\|=\|\cdot\|_{\left(A_{0}, A_{1}\right)_{1 / 2,2}}$; in particular, under the hypotheses of Lemma 3, we have the following obvious corollary. 
Corollary 1. Under the assumption of Lemma 3 and setting

$$
\|\cdot\|:=\left(\|\cdot\|_{V}^{2}+\left\|\mathcal{L}_{\text {skew }} \cdot\right\|_{\left(C_{0}, C_{1}\right)_{1 / 2,2}}^{2}\right)^{1 / 2},
$$

we have the continuity and inf-sup conditions (3)-(4) for $\mathcal{L}$, with constants $\mathcal{C}_{c}$ and $\mathcal{C}_{i s}$ independent of $\mathcal{L}$.

Actually Lemma 2 establishes a family of continuity and inf-sup conditions for $\mathcal{L}$ (for different values of $\theta$ and $p$ ) with different norms on the trial space (i.e., $\|\cdot\|_{\left(A_{0}, A_{1}\right)_{\theta, p}}$ ) and on the test space (i.e., $\|\cdot\|_{\left.\left(A_{0}, A_{1}\right)_{1-\theta, p^{\prime}}\right)}$; on the other hand from the numerical standpoint (3)-(4) are mainly interesting, as discussed in $[17, \S 4]$.

\section{The convection-diffusion-reaction operator}

We now apply the results of the previous section to the convection-diffusion-reaction operator. In Lemma 1-3 we have explicitly computed the constants involved into the estimates, in order to emphasize that the estimates do not depend on $\mathcal{L}$; henceforth, for the sake of simplicity, we will use generic constants denoted by $\mathcal{C}, \mathcal{C}_{1}, \mathcal{C}_{2}$, which are independent on the operator coefficients $\kappa$, $\beta$ and $\rho$ and on the domain $\Omega$.

\subsection{The one-dimensional case}

We start with the analysis of the very simple one-dimensional operator, with constant coefficients $\kappa>0$ and $\rho \geq 0$, and unitary velocity. Then, for this subsection only, we will consider a special case of (1), which is

$$
w \mapsto \mathcal{L} w:=-\kappa w^{\prime \prime}+w^{\prime}+\rho w,
$$

where the argument $w$ is a function on the interval $\Omega=[0,1]$.

We consider first, and with particular emphasis, the ordinary differential equation with homogeneous Dirichlet boundary conditions (2). The variational formulation (6) reads

$$
\text { find } u \in V \text { such that } a(u, v)=\int_{0}^{1} f v, \forall v \in V \text {, }
$$

where

$$
\begin{gathered}
V=H_{0}^{1}(0,1) \text { with }\|\cdot\|_{V}^{2}=\kappa|\cdot|_{H^{1}}^{2}+\rho\|\cdot\|_{L^{2}}^{2}, \\
a(w, v)=\kappa \int_{0}^{1} w^{\prime} v^{\prime}+\int_{0}^{1} w^{\prime} v+\rho \int_{0}^{1} w v .
\end{gathered}
$$

Then $\mathcal{L}_{\text {sym }} w=-\kappa w^{\prime \prime}+\rho w, \mathcal{L}_{\text {skew }} w=w^{\prime}, a_{\text {sym }}(w, v)=\kappa \int_{0}^{1} w^{\prime} v^{\prime}+\rho \int_{0}^{1} w v$ and $a_{\text {skew }}(w, v)=\int_{0}^{1} w^{\prime} v$. Finally $C_{0}=L_{0}^{2}(0,1)$ and $C_{1}=H^{-1}(0,1)$ from the algebraic standpoint, where $L_{0}^{2}$ is the subspace of $L^{2}$ of zero mean value functions, and its natural norm is $\|\cdot\|_{L_{0}^{2}}:=\|\cdot\|_{L^{2}}$, while $H^{-1}$ is the dual of $H_{0}^{1}$, endowed with the dual norm $\|\cdot\|_{H^{-1}}=\sup _{v \in H_{0}^{1}(0,1)}\langle\cdot, v\rangle /|v|_{H^{1}}$ (we recall that 
$|\cdot|_{H^{1}}:=\left[\int_{0}^{1}\left(w^{\prime}\right)^{2}\right]^{1 / 2}$ is a norm on $\left.H_{0}^{1}\right)$. It is easy to see that $L_{0}^{2}$ is a dense subspace of $H^{-1}$. From Corollary 1 we immediately have the following result.

Theorem 1. For the case (31)-(32), uniform continuity and inf-sup conditions (3)-(4) hold true with respect to the norm

$$
w \mapsto\|w\|=\left(\kappa|w|_{H^{1}}^{2}+\left\|w^{\prime}\right\|_{\left(C_{0}, C_{1}\right)_{1 / 2,2}}^{2}+\rho\|w\|_{L^{2}}^{2}\right)^{1 / 2}
$$

Now we focus our attention on $\|\cdot\|$ in (33), in order to better understand its structure. Roughly speaking, the term $\left\|w^{\prime}\right\|_{\left(C_{0}, C_{1}\right)_{1 / 2,2}}$ is related to the skew-symmetric part of $\mathcal{L}$, which is the first order derivative. Then we expect $w \mapsto\left\|w^{\prime}\right\|_{\left(C_{0}, C_{1}\right)_{1 / 2,2}}$ to act as a 1/2-order norm uniformly on the operator coefficients $\kappa$ and $\rho$. That is in fact stated in the next theorem: we show that $\left\|w^{\prime}\right\|_{\left(C_{0}, C_{1}\right)_{1 / 2,2}}$ stays between the $H^{1 / 2}$-seminorm and $H_{00}^{1 / 2}$-norm, where $H^{1 / 2}:=\left(L^{2}, H^{1}\right)_{1 / 2,2}$ and $H_{00}^{1 / 2}:=\left(L^{2}, H_{0}^{1}\right)_{1 / 2,2}$ are the two usual Hilbert spaces of order $1 / 2$, endowed with the usual norms given by interpolation (see [15]), and $|w|_{H^{1 / 2}}$ is the seminorm $\left\|w-\Pi_{0} w\right\|_{H^{1 / 2}}$, $\Pi_{0}$. denoting the mean value of its argument.

Theorem 2. For the case (31)-(32), we have

$$
\mathcal{C}_{1}|w|_{H^{1 / 2}} \leq\left\|w^{\prime}\right\|_{\left(C_{0}, C_{1}\right)_{1 / 2,2}} \leq \mathcal{C}_{2}\|w\|_{H_{00}^{1 / 2}}, \quad \forall w \in V
$$

Proof. When $\rho=0$, (34) follows from (48); we assume henceforth $\rho>0$.

We consider first the left inequality in (34), i.e.

$$
\mathcal{C}|w|_{H^{1 / 2}} \leq\left\|w^{\prime}\right\|_{\left(C_{0}, C_{1}\right)_{1 / 2,2}}, \quad \forall w \in V .
$$

It is easy to see that $\left\|z^{\prime}\right\|_{L^{2}} \simeq\|z\|_{H^{1}}$ and $\left\|z^{\prime}\right\|_{H^{-1}} \simeq\|z\|_{L^{2}}$, for any $z \in H^{1} \cap L_{0}^{2}$; then, thanks to Theorem $[19, \S 1.3 .3],[19, \S 1.11 .2]$ and $[19, \S 1.17 .1]$, the first order derivative is a topological isomorphism from $H^{1 / 2} \cap L_{0}^{2}$ into $\left(H^{-1}, L^{2}\right)_{1 / 2,2}$, which means

$$
|w|_{H^{1 / 2}}=\left\|w-\Pi_{0} w\right\|_{H^{1 / 2}} \simeq\left\|w^{\prime}\right\|_{\left(H^{-1}, L^{2}\right)_{1 / 2,2}} .
$$

We introduce now the new space $\widetilde{C}_{0}$ : from the algebraic standpoint we set $\widetilde{C}_{0}:=L^{2}$, and we define $\|\cdot\|_{\widetilde{C}_{0}}:=\left(\kappa\|\cdot\|_{L^{2}}^{2}+\rho\|\cdot\|_{H^{-1}}^{2}\right)^{1 / 2}$. Our next step is to show that

$$
\|\phi\|_{\left(H^{-1}, L^{2}\right)_{1 / 2,2}} \leq \mathcal{C}\|\phi\|_{\left(\widetilde{C}_{0}, C_{1}\right)_{1 / 2,2}}, \quad \forall \phi \in L^{2}
$$

For that purpose we split a generic $\phi \in L^{2}$ into

$$
\phi=\phi_{\text {high }}+\phi_{\text {low }}
$$

where $\phi_{\text {high }}, \phi_{\text {low }} \in L^{2}$ are, roughly speaking, the high frequency part and the low frequency part of $\phi$, respectively, in such a way that

$$
\begin{gathered}
\kappa^{1 / 2}\left\|\phi_{\text {high }}\right\|_{L^{2}}+\rho^{1 / 2}\left\|\phi_{\text {low }}\right\|_{H^{-1}} \leq \mathcal{C}\|\phi\|_{\widetilde{C}_{0}} \\
\kappa^{-1 / 2}\left\|\phi_{\text {high }}\right\|_{H^{-1}}+\rho^{-1 / 2}\left\|\phi_{\text {low }}\right\|_{L^{2}} \leq \mathcal{C}\|\phi\|_{C_{1}} .
\end{gathered}
$$


For that purpose, we introduce an auxiliary problem: let $\psi \in H_{0}^{1}$ the solution of

$$
\mathcal{L}_{\text {sym }} \psi=\phi \quad \text { in }(0,1)
$$

and let $\phi_{\text {high }}:=-\kappa \psi^{\prime \prime}$ and $\phi_{\text {low }}:=\rho \psi$.

Multiplying both members of the differential equation $(41)$ by $-\psi^{\prime \prime}$, integrating over $(0,1)$ and integrating by parts we get

$$
\kappa\left\|\psi^{\prime \prime}\right\|_{L^{2}}^{2}+\rho\left\|\psi^{\prime}\right\|_{L^{2}}^{2}=-\int_{0}^{1} \phi \psi^{\prime \prime} ;
$$

then, thanks to the Cauchy-Schwartz inequality, we have

$$
\left\|\phi_{\text {high }}\right\|_{L^{2}}=\left\|\kappa \psi^{\prime \prime}\right\|_{L^{2}} \leq\|\phi\|_{L^{2}} .
$$

Integrating (41) we have

$$
-\kappa \psi^{\prime}+\kappa \psi^{\prime}(0)+\rho \Psi=\Phi
$$

where $\Psi(x)=\int_{0}^{x} \psi(t) d t$ and analogously $\Phi(x)=\int_{0}^{x} \phi(t) d t$; after multiplying by $\Psi-\Pi_{0} \Psi$ both members, integrating over $(0,1)$ and integrating by parts we obtain

$$
\kappa\|\psi\|_{L^{2}}^{2}+\rho\left\|\Psi-\Pi_{0} \Psi\right\|_{L^{2}}^{2}=\int_{0}^{1} \Phi\left(\Psi-\Pi_{0} \Psi\right),
$$

whence now

$$
\left\|\phi_{\text {low }}\right\|_{H^{-1}}=\rho\left\|\Psi-\Pi_{0} \Psi\right\|_{L^{2}} \leq\left\|\Phi-\Pi_{0} \Phi\right\|_{L^{2}}=\|\phi\|_{H^{-1}} .
$$

Collecting (42)-(43) we obtain (39). From (41) it is also easy to obtain the estimate $\left(\kappa\left\|\psi^{\prime}\right\|_{L^{2}}^{2}+\right.$ $\left.\rho\|\psi\|_{L^{2}}^{2}\right)^{1 / 2} \leq\|\phi\|_{V^{*}}=\|\phi\|_{C_{1}}$, which gives (40) straightforwardly.

Consider now the linear operator $\phi \mapsto\left(\phi_{\text {high }}, \phi_{\text {low }}\right)$ from $L^{2}$ into $L^{2} \times L^{2}$, with $\phi_{\text {high }}$, $\phi_{\text {low }}$ as defined above: by interpolation from the two continuity estimates (39)-(40) we get

$$
\left\|\phi_{\text {high }}\right\|_{\left(L^{2}, H^{-1}\right)_{1 / 2,2}}+\left\|\phi_{\text {low }}\right\|_{\left(H^{-1}, L^{2}\right)_{1 / 2,2}} \leq \mathcal{C}\|\phi\|_{\left(\widetilde{C}_{0}, C_{1}\right)_{1 / 2,2}}
$$

whence, by using the triangle inequality and since $\|\cdot\|_{\left(L^{2}, H^{-1}\right)_{1 / 2,2}}=\|\cdot\|_{\left(H^{-1}, L^{2}\right)_{1 / 2,2}}$, we obtain (37). Finally (36) and (37) gives (35).

Now we consider the right equivalence in (34), which is

$$
\left\|w^{\prime}\right\|_{\left(C_{0}, C_{1}\right)_{1 / 2,2}} \leq \mathcal{C}\|w\|_{H_{00}^{1 / 2}}, \quad \forall w \in V .
$$

Given $w \in H_{0}^{1}$ it is easy to see that

$$
\left\|w^{\prime}\right\|_{C_{0}}=\|w\|_{V}=\|w\|_{C_{1}^{*}},
$$

and

$$
\left\|w^{\prime}\right\|_{C_{1}}=\left\|w^{\prime}\right\|_{V^{*}} \leq\|w\|_{\widetilde{C}_{0}^{*}}
$$


whence (thanks to Theorem $[19, \S 1.11 .2]$ )

$$
\left\|w^{\prime}\right\|_{\left(C_{0}, C_{1}\right)_{1 / 2,2}} \leq\|w\|_{\left(C_{1}^{*}, \widetilde{C}_{0}^{*}\right)_{1 / 2,2}}=\|w\|_{\left(\widetilde{C}_{0}, C_{1}\right)_{1 / 2,2}^{*}}
$$

Moreover, passing to the duals in (37),still using Theorem $[19, \S 1.11 .2]$, we also have

$$
\|w\|_{\left(\widetilde{C}_{0}, C_{1}\right)_{1 / 2,2}^{*}} \leq\|w\|_{\left(H^{-1}, L^{2}\right)_{1 / 2,2}^{*}}=\|w\|_{\left(H^{1}, L^{2}\right)_{1 / 2,2}}=\|w\|_{\left(H_{00}^{1 / 2}\right)} .
$$

Inequalities (46)-(47) give (45).

Remark 1. It is worth noting that Theorem 1-2 allow for $\rho=0$ as well; in that case we have $\left\|w^{\prime}\right\|_{\left(C_{0}, C_{1}\right)_{1 / 2,2}}=\left\|w^{\prime}\right\|_{\left(H^{-1}, L_{0}^{2}\right)_{1 / 2,2}}$, since the coefficient $\kappa$ easily cancel when interpolating. Let $H_{\#}^{1}$ be the subspace of $H^{1}$ of functions $w$ such that $w(0)=w(1)$, endowed with the $\|\cdot\|_{H_{\#}^{1}}:=\|\cdot\|_{H^{1}}$, and $H_{\#}^{1 / 2}:=\left(L^{2}, H_{\#}^{1}\right)_{1 / 2,2}$ endowed with the norm given by interpolation. Given $z \in H_{\#}^{1} \cap L_{0}^{2}$, one has $\left\|z^{\prime}\right\|_{L_{0}^{2}} \simeq\|z\|_{H_{\#}^{1}}$ and $\left\|z^{\prime}\right\|_{H^{-1}} \simeq\|z\|_{L^{2}}$, whence (by using Theorem [19, §1.3.3], [19, §1.11.2] and [19, §1.17.1]) $\left\|z^{\prime}\right\|_{\left(H^{-1}, L_{0}^{2}\right)_{1 / 2,2}} \simeq\|z\|_{\left(L^{2}, H_{\#}^{1}\right)_{1 / 2,2}}$ and therefore $\left\|w^{\prime}\right\|_{\left(H^{-1}, L_{0}^{2}\right)_{1 / 2,2}} \simeq\left\|w-\Pi_{0} w\right\|_{\left(L^{2}, H_{\#}^{1}\right)_{1 / 2,2}}$, for any $w \in H_{0}^{1}$; this means that we have the following characterization:

$$
\rho=0 \Rightarrow|w|_{H_{\#}^{1 / 2}}:=\left\|w-\Pi_{0} w\right\|_{\left(L^{2}, H_{\#}^{1}\right)_{1 / 2,2}}=\left\|w^{\prime}\right\|_{\left(C_{0}, C_{1}\right)_{1 / 2,2}}, \quad \forall w \in V .
$$

We may also deal with different kind of boundary conditions; consider the example

$$
\left\{\begin{aligned}
\mathcal{L} u & =f \quad \text { in }(0,1) \\
u(0) & =u^{\prime}(1)=0,
\end{aligned}\right.
$$

where $\mathcal{L}$ is still formally given by (31). The variational formulation (6) now requires

$$
\begin{aligned}
& V=\left\{v \in H^{1}(0,1) \text { such that } v(0)=0\right\} \\
& a(w, v)=\kappa \int_{0}^{1} w^{\prime} v^{\prime}+\int_{0}^{1} w^{\prime} v+\rho \int_{0}^{1} w v
\end{aligned}
$$

the key point is that the bilinear form $a(\cdot, \cdot)$ is coercive on $V$; accordingly, we define $\|\cdot\|_{V}$ as

$$
\|w\|_{V}^{2}:=a(w, w)=\kappa|w|_{H^{1}}^{2}+\rho\|w\|_{L^{2}}^{2}+\frac{1}{2} w(1)^{2}
$$

and we have now

$$
\begin{gathered}
a_{\mathrm{sym}}(w, v)=\kappa \int_{0}^{1} w^{\prime} v^{\prime}+\rho \int_{0}^{1} w v+\frac{1}{2} w(1) v(1), \\
a_{\text {skew }}(w, v)=\int_{0}^{1} w^{\prime} v-\frac{1}{2} w(1) v(1) .
\end{gathered}
$$

Then we can still make use of the theory of $\S 2$ and obtain uniform inf-sup and continuity conditions from Corollary 1. 
When the bilinear form $a(\cdot, \cdot)$ is not coercive, then we can not use the results of $\S 2$. This is the case of

$$
\left\{\begin{aligned}
-\kappa u^{\prime \prime}+u^{\prime} & =f \quad \text { in }(0,1) \\
u^{\prime}(0)=u(1) & =0
\end{aligned}\right.
$$

i.e., when $\rho=0$ and we prescribe Neumann boundary condition at the inflow $x=0$; then $V=\left\{v \in H^{1}(0,1)\right.$ such that $\left.v(1)=0\right\}$ and

$$
a(w, w)=\kappa|w|_{H^{1}}^{2}+\rho\|w\|_{L^{2}}^{2}-\frac{1}{2} w(1)^{2}
$$

which is not positive in general, when $\kappa$ and $\rho$ are small enough. However, when $f=1$ the solution of $(50)$ is $u(x)=\kappa(\exp (1 / \kappa)-\exp (x / \kappa))+x-1$; for $\kappa \rightarrow 0$ we have $\|u\|_{L^{2}} \approx \kappa \exp (1 / \kappa)$, whence we see that (50) is in fact not uniformly well posed with respect to $\kappa$.

\subsection{The multi-dimensional case}

In this section, we analyze the multi-dimensional convection-diffusion-reaction operator with Dirichlet homogeneous boundary conditions (1)-(2), and the associated bilinear form

$$
a(w, v)=\kappa \int_{\Omega} \nabla w \cdot \nabla v+\int_{\Omega} \beta \cdot \nabla w v+\int_{\Omega} \rho w v
$$

which is defined on $H_{0}^{1}(\Omega) \times H_{0}^{1}(\Omega)$ (see, e.g., [15]). Under the assumption

$$
\rho-\frac{1}{2} \operatorname{div}(\beta) \geq 0
$$

the bilinear form $a(\cdot, \cdot)$ is coercive, whence we set

$$
\begin{gathered}
V=H_{0}^{1}(\Omega) \\
\|w\|_{V}^{2}=a(w, w)=\kappa|w|_{H^{1}}^{2}+\left(\rho-\frac{1}{2} \operatorname{div}(\beta)\right)\|w\|_{L^{2}}^{2} .
\end{gathered}
$$

The decomposition (8) gives

$$
\begin{aligned}
& a_{\text {sym }}(w, v)=\kappa \int_{\Omega} \nabla w \cdot \nabla v+\int_{\Omega}\left(\rho-\frac{1}{2} \operatorname{div}(\beta)\right) w v \\
& a_{\text {skew }}(w, v)=\int_{\Omega} \beta \cdot \nabla w v+\frac{1}{2} \int_{\Omega} \operatorname{div}(\beta) w v
\end{aligned}
$$

For the sake of simplicity, we shall consider henceforth the case

$$
\operatorname{div}(\beta)=0
$$


In order to apply Corollary 1 to this case, we need $\mathcal{L}_{\text {skew }}=\beta \cdot \nabla$ to be injective on $V$ : this is assured, for example, by the assumption

$$
\text { there exists a smooth } \phi: \Omega \rightarrow \mathbb{R} \text { such that } \nabla \phi \cdot \beta \geq \mathcal{C}>0 \text {; }
$$

we refer to [14] for further details. Definition (24) says that, from the algebraic standpoint, $C_{0}$ is the space of the streamline derivatives $\beta \cdot \nabla w$ of functions $w \in H_{0}^{1}$, while $C_{1}$ is $H^{-1}$. Corollary 1 gives then the following result

Theorem 3. For the case (52), (54)-(55), the uniform continuity and inf-sup conditions (3)-(4) hold true with respect to the norm

$$
w \mapsto\|w\|=\left(\kappa|w|_{H^{1}}^{2}+\|\beta \cdot \nabla w\|_{\left(C_{0}, C_{1}\right)_{1 / 2,2}}^{2}+\rho\|w\|_{L^{2}}^{2}\right)^{1 / 2} .
$$

Roughly speaking, we expect $\|\beta \cdot \nabla w\|_{\left(C_{0}, C_{1}\right)_{1 / 2,2}}$ to be of order $1 / 2$ in the direction of $\beta$, and of order 0 in the directions orthogonal to $\beta$ (this can be more easily seen for the case $\rho=0$ ), but a rigorous analysis of the structure of $\|\beta \cdot \nabla w\|_{\left(C_{0}, C_{1}\right)_{1 / 2,2}}$ is more difficult now than for the simpler one-dimensional case considered in $\S 3.1$. The next result shows that $\|\beta \cdot \nabla w\|_{\left(C_{0}, C_{1}\right)_{1 / 2,1}}$ has some uniform bounds independent of $\kappa$ and $\rho$ (though the anisotropy is not investigated). Then we end by a comparison between $\|\beta \cdot \nabla w\|_{\left(C_{0}, C_{1}\right)_{1 / 2,1}}$ and $\|\beta \cdot \nabla w\|_{\left(C_{0}, C_{1}\right)_{1 / 2,2}}$.

Proposition 1. For the case (52), (54)-(55), we have:

$$
\mathcal{C}_{p}\|\beta\|_{L^{\infty}}^{1 / 2} \operatorname{diam}(\Omega)^{-1 / 2}\|w\|_{L^{2}} \leq\|\beta \cdot \nabla w\|_{\left(C_{0}, C_{1}\right)_{1 / 2,1}} \leq \mathcal{C}\|\beta\|_{L^{\infty}}^{1 / 2}\|w\|_{\left(L^{2}, H_{0}^{1}\right)_{1 / 2,1}}, \quad \forall w \in V,
$$

where the constant $\mathcal{C}_{p}$ of the Poincaré-like inequality depends on $\beta /\|\beta\|_{L^{\infty}}$ and (the shape of) $\Omega$.

Proof. Let $\eta$ be the solution of $\beta \cdot \nabla \eta=\|\beta\|_{L^{\infty}}$ with $\eta=0$ on $\partial \Omega^{-}:=\{\mathbf{x} \in \partial \Omega \mid \beta(\mathbf{x}) \cdot \mathbf{n}(\mathbf{x})<0\}, \mathbf{n}$ denoting the outward normal unit vector defined on $\partial \Omega$; the existence of $\eta$ is guaranteed by (55). Given $w \in H_{0}^{1}$, integrating by parts, using the Cauchy-Schwartz inequality and (54) we have

$$
\begin{aligned}
\|\beta\|_{L^{\infty}}\|w\|_{L^{2}}^{2} & =\int_{\Omega} \beta \cdot \nabla \eta w^{2} \\
& =-2 \int_{\Omega} \eta w \beta \cdot \nabla w \\
& \leq 2\|\eta w\|_{V}\|\beta \cdot \nabla w\|_{V^{*}}
\end{aligned}
$$

We have

$$
\|\eta w\|_{L^{2}} \leq\|\eta\|_{L^{\infty}}\|w\|_{L^{2}}
$$

and, using the classical Poincaré inequality, it is easy to get

$$
\begin{aligned}
|\eta w|_{H^{1}} & \leq \mathcal{C}\left(\|\eta\|_{L^{\infty}}|w|_{H^{1}}+\|\nabla \eta\|_{\left(L^{\infty}\right)^{2}}\|w\|_{L^{2}}\right) \\
& \leq \mathcal{C}\left(\|\eta\|_{L^{\infty}}+\operatorname{diam}(\Omega)\|\nabla \eta\|_{\left(L^{\infty}\right)^{2}}\right)|w|_{H^{1}}
\end{aligned}
$$


Moreover, thanks to (55), we have $\widetilde{\mathcal{C}_{p}}:=\operatorname{diam}(\Omega)^{-1}\|\eta\|_{L^{\infty}}+\|\nabla \eta\|_{\left(L^{\infty}\right)^{2}}<+\infty$ (e.g., see [14, Theorem 3.2]), where $\widetilde{\mathcal{C}_{p}}$ depends on $\eta$, i.e. on $\beta /\|\beta\|_{L^{\infty}}$ and on (the shape of) $\Omega$. Then

$$
\|\eta w\|_{V} \leq \mathcal{C} \widetilde{\mathcal{C}_{p}} \operatorname{diam}(\Omega)\|w\|_{V}
$$

substituting back in (58),

$$
\begin{aligned}
\|\beta\|_{L^{\infty}}\|w\|_{L^{2}}^{2} & \leq \mathcal{C} \widetilde{\mathcal{C}_{p}} \operatorname{diam}(\Omega)\|w\|_{V}\|\beta \cdot \nabla w\|_{V^{*}} \\
& =\mathcal{C} \widetilde{\mathcal{C}_{p}} \operatorname{diam}(\Omega)\|\beta \cdot \nabla w\|_{C_{0}}\|\beta \cdot \nabla w\|_{C_{1}},
\end{aligned}
$$

and thanks to Theorem $[19, \S 1.10 .1]$ we obtain

$$
\mathcal{C}_{p}\|\beta\|_{L^{\infty}}^{1 / 2} \operatorname{diam}(\Omega)^{-1 / 2}\|w\|_{L^{2}} \leq\|\beta \cdot \nabla w\|_{\left(C_{0}, C_{1}\right)_{1 / 2,1}}, \quad \forall w \in V
$$

which is the left inequality of (57).

We have, thanks to Theorem $[19, \S 1.3 .3]$

$$
\begin{aligned}
\|\beta \cdot \nabla w\|_{\left(C_{0}, C_{1}\right)_{1 / 2,1}}^{2} \leq & \|\beta \cdot \nabla w\|_{C_{0}}\|\beta \cdot \nabla w\|_{C_{1}} \\
\leq & \kappa^{1 / 2}|w|_{H^{1}}\|\beta \cdot \nabla w\|_{V^{*}} \\
& +\rho^{1 / 2}\|w\|_{L^{2}}\|\beta \cdot \nabla w\|_{V^{*}}
\end{aligned}
$$

and

$$
\begin{gathered}
\|\beta \cdot \nabla w\|_{V^{*}} \leq \kappa^{-1 / 2}\|\beta \cdot \nabla w\|_{H^{-1}} \leq \kappa^{-1 / 2}\|\beta\|_{L^{\infty}}\|w\|_{L^{2}}, \\
\|\beta \cdot \nabla w\|_{V^{*}} \leq \rho^{-1 / 2}\|\beta \cdot \nabla w\|_{L^{2}} \leq \rho^{-1 / 2}\|\beta\|_{L^{\infty}}|w|_{H^{1}}
\end{gathered}
$$

from (62)-(63), we get

$$
\|\beta \cdot \nabla w\|_{\left(C_{0}, C_{1}\right)_{1 / 2,1}}^{2} \leq 2\|\beta\|_{L^{\infty}}|w|_{H^{1}}\|w\|_{L^{2}},
$$

and Theorem [19, §1.10.1] yields

$$
\|\beta \cdot \nabla w\|_{\left(C_{0}, C_{1}\right)_{1 / 2,1}} \leq \mathcal{C}\|\beta\|_{L^{\infty}}^{1 / 2}\|w\|_{\left(L^{2}, H_{0}^{1}\right)_{1 / 2,1}}, \quad \forall w \in V,
$$

and concludes the proof of (57).

In the previous proposition, we have shown uniform bounds (with respect to the operator coefficients) for $\|\beta \cdot \nabla w\|_{\left(C_{0}, C_{1}\right)_{1 / 2,1}}$; as a general result of the interpolation theory (see, e.g., [19, 1.3.3.d]), we have

$$
\|\beta \cdot \nabla w\|_{\left(C_{0}, C_{1}\right)_{1 / 2,2}} \leq \mathcal{C}\|\beta \cdot \nabla w\|_{\left(C_{0}, C_{1}\right)_{1 / 2,1}}, \quad \forall w \in V
$$

and similarly

$$
\|w\|_{\left(A_{0}, A_{1}\right)_{1 / 2,2}} \leq \mathcal{C}\|w\|_{\left(A_{0}, A_{1}\right)_{1 / 2,1}}, \quad \forall w \in V ;
$$

the converse inequality of $(64)$, that is $\|\beta \cdot \nabla w\|_{\left(C_{0}, C_{1}\right)_{1 / 2,1}} \leq \mathcal{C}\|\beta \cdot \nabla w\|_{\left(C_{0}, C_{1}\right)_{1 / 2,2}}$, does not hold true; on the other hand the converse of (65) holds true, and it is, roughly speaking, almost uniform, in the sense that the constant in it only depends on a logarithm of the coefficients, as stated in the next proposition. 
Proposition 2. Consider the case (52), (54) and (55); let

$$
\alpha:=\max \left\{\kappa^{1 / 2} \rho^{1 / 2}, \kappa \operatorname{diam}(\Omega)\right\} /\|\beta\|_{L^{\infty}} .
$$

When $\alpha \leq 1$ we have

$$
\|w\|_{\left(A_{0}, A_{1}\right)_{1 / 2,1}} \leq\left(\mathcal{C}-\log ^{1 / 2}(\alpha)\right)\|w\|_{\left(A_{0}, A_{1}\right)_{1 / 2,2}}, \quad \forall w \in V
$$

while for $\alpha>1$ we have

$$
\|w\|_{\left(A_{0}, A_{1}\right)_{1 / 2,1}} \leq \mathcal{C}\|w\|_{\left(A_{0}, A_{1}\right)_{1 / 2,2},}, \quad \forall w \in V
$$

Proof. We only consider here the case $\alpha \leq 1$, since when $\alpha>1$ we can set $\alpha:=1$ instead of (66) and follow the proof. First, recall that from the definition (10) we have

$$
\begin{aligned}
& \|w\|_{A_{0}} \leq\|w\|_{A_{0}}, \quad \forall w \in V, \\
& \|w\|_{A_{0}} \leq\|w\|_{A_{1}}, \quad \forall w \in V,
\end{aligned}
$$

and, since (63) and the Poincaré inequality, we also have

$$
\begin{aligned}
\|w\|_{A_{1}} & \leq\|w\|_{A_{1}}, \quad \forall w \in V, \\
\alpha\|w\|_{A_{1}} & \leq \mathcal{C}\|w\|_{A_{0}}, \quad \forall w \in V,
\end{aligned}
$$

then, by interpolation, we get from (69)

$$
\|w\|_{A_{0}} \leq\|w\|_{\left(A_{0}, A_{1}\right)_{1 / 2,2}}, \quad \forall w \in V
$$

and from (70)

$$
\alpha^{1 / 2}\|w\|_{A_{1}} \leq \mathcal{C}\|w\|_{\left(A_{0}, A_{1}\right)_{1 / 2,2}}, \quad \forall w \in V .
$$

By the definition (19) and by the triangle inequality we get

$$
\begin{aligned}
\|w\|_{\left(A_{0}, A_{1}\right)_{1 / 2,1} \leq} & \int_{0}^{+\infty}\left(t^{-1 / 2}\left\|w_{0}(t)\right\|_{A_{0}}+t^{1 / 2}\left\|w_{1}(t)\right\|_{A_{1}}\right) \frac{d t}{t} \\
\leq & \int_{0}^{\alpha}\left(t^{-1 / 2}\left\|w_{0}(t)\right\|_{A_{0}}+t^{1 / 2}\left\|w_{1}(t)\right\|_{A_{1}}\right) \frac{d t}{t} \\
& +\int_{\alpha}^{1}\left(t^{-1 / 2}\left\|w_{0}(t)\right\|_{A_{0}}+t^{1 / 2}\left\|w_{1}(t)\right\|_{A_{1}}\right) \frac{d t}{t} \\
& +\int_{1}^{+\infty}\left(t^{-1 / 2}\left\|w_{0}(t)\right\|_{A_{0}}+t^{1 / 2}\left\|w_{1}(t)\right\|_{A_{1}}\right) \frac{d t}{t} \\
= & I+I I+I I I,
\end{aligned}
$$


for any $w_{0}(t)$ and $w_{1}(t)$ with $w=w_{0}(t)+w_{1}(t), w_{i}(t) \in V, i=1,2$ and $0<t<+\infty$. Taking $w_{0}(t)=w$ and $w_{1}(t)=0$ for $t \geq 1$, and using (71) we have

$$
\begin{aligned}
I I I & \leq\|w\|_{A_{0}} \int_{1}^{\infty} t^{-3 / 2} d t \\
& \leq 2\|w\|_{A_{0}} \\
& \leq 2\|w\|_{\left(A_{0}, A_{1}\right)_{1 / 2,2}} .
\end{aligned}
$$

In a very similar way, we deal with the first term, taking $w_{1}(t)=w$ and $w_{0}(t)=0$ for $0<t<\alpha$; thanks to (72) we obtain:

$$
\begin{aligned}
I & \leq\|w\|_{A_{1}} \int_{0}^{\alpha} t^{-1 / 2} d t \\
& \leq 2 \alpha^{1 / 2}\|w\|_{A_{1}} \\
& \leq \mathcal{C}\|w\|_{\left(A_{0}, A_{1}\right)_{1 / 2,2}} .
\end{aligned}
$$

Thanks to the Cauchy-Schwartz inequality we have

$$
\begin{aligned}
\int_{\alpha}^{1}\left(t^{-1 / 2}\left\|w_{0}(t)\right\|_{A_{0}}+t^{1 / 2}\left\|w_{1}(t)\right\|_{A_{1}}\right) \frac{d t}{t} \\
\leq\left[\int_{\alpha}^{1} \frac{d t}{t}\right]^{1 / 2} \\
\cdot\left[\int_{\alpha}^{1}\left(t^{-1 / 2}\left\|w_{0}(t)\right\|_{A_{0}}+t^{1 / 2}\left\|w_{1}(t)\right\|_{A_{1}}\right)^{2} \frac{d t}{t}\right]^{1 / 2} \\
\leq[-\log (\alpha)]^{1 / 2} \\
\cdot\left[\int_{\alpha}^{1}\left(t^{-1 / 2}\left\|w_{0}(t)\right\|_{A_{0}}+t^{1 / 2}\left\|w_{1}(t)\right\|_{A_{1}}\right)^{2} \frac{d t}{t}\right]^{1 / 2}
\end{aligned}
$$

that holds true for any choice of $w_{0}(t)$ and $w_{1}(t)$ on $\alpha<t<1$; taking the infimum on $w_{0}$, $w_{1}$ we obtain

$$
I I \leq[-\log (\alpha)]^{1 / 2}\|w\|_{\left(A_{0}, A_{1}\right)_{1 / 2,2}} .
$$

Finally, (67) follows from the previous estimates on $I, I I$ and $I I I$.

From Proposition 1-2 we easily derive the next almost uniform bounds (still, up to a $\log (\alpha)^{1 / 2}$ factor, which is, roughly speaking, a weak loss of uniformity).

Corollary 2. For the case (52), (54)-(55), given a from (66), we have:

$$
\begin{gathered}
\mathcal{C}_{p} \min \left\{1,|\log (\alpha)|^{-1 / 2}\right\} \operatorname{diam}(\Omega)^{-1 / 2}\|\beta\|_{L^{\infty}}^{1 / 2}\|w\|_{L^{2}} \leq\|w\|, \quad \forall w \in V, \\
\|\beta \cdot \nabla w\|_{\left(C_{0}, C_{1}\right)_{1 / 2,2}} \leq \mathcal{C}\|\beta\|_{L^{\infty}}^{1 / 2}\|w\|_{\left(L^{2}, H_{0}^{1}\right)_{1 / 2,1}}, \quad \forall w \in V .
\end{gathered}
$$

where $\mathcal{C}_{p}$ depends on $\beta /\|\beta\|_{L^{\infty}}$ and (the shape of) $\Omega$. 
Though (74)-(75) are not sharp estimates as we got in $\S 3.1$ for the one-dimensional case, they put in evidence the relationship between the norm $\|\cdot\|$ defined in (56), and the skew-symmetric part $\mathcal{L}_{\text {skew }}=\beta \cdot \nabla$ of (1). Recall that $\max \left\{\kappa^{1 / 2} \operatorname{diam}(\Omega)^{-1}, \rho^{1 / 2}\right\}\|w\|_{L^{2}} \leq \mathcal{C}\|w\|_{V} \leq \mathcal{C}\|w\|$, while (74) states the bound on the $L^{2}$-norm which is mainly due to $\|\beta \cdot \nabla w\|_{\left(C_{0}, C_{1}\right)_{1 / 2,2}}$. Then (74) becomes relevant when $\kappa$ and $\rho$ are small.

\section{References}

[1] I. BABUŠKA AND A. K. AzIZ, Survey lectures on the mathematical foundations of the finite element method, in The mathematical foundations of the finite element method with applications to partial differential equations (Proc. Sympos., Univ. Maryland, Baltimore, Md., 1972), Academic Press, New York, 1972, pp. 1-359. With the collaboration of G. Fix and R. B. Kellogg.

[2] A. Barinka, T. Barsch, P. Charton, A. Cohen, S. Dahlke, W. Dahmen, and K. URBAn, Adaptive wavelet schemes for elliptic problems - implementation and numerical experiments, SIAM J. Sci. Comput., 23 (2001), pp. 910-939 (electronic).

[3] S. Berrone And C. CAnuto, Multilevel a posteriori error analysis for reaction-convectiondiffusion problems, Tech. Rep. 18/2002, Dipartimento di Matematica, Politecnico di Torino, 2002 .

[4] S. Bertoluzza, C. Canuto, And A. Tabacco, Stable discretizations of convectiondiffusion problems via computable negative-order inner products, SIAM J. Numer. Anal., 38 (2000), pp. 1034-1055 (electronic).

[5] S. Bertoluzza And M. Verani, Convergence of a nonlinear wavelet algorithm for the solution of PDEs, Appl. Math. Lett., 16 (2003), pp. 113-118.

[6] J. H. Bramble, R. D. Lazarov, and J. E. Pasciak, Least-squares for second-order elliptic problems, Comput. Methods Appl. Mech. Engrg., 152 (1998), pp. 195-210. Symposium on Advances in Computational Mechanics, Vol. 5 (Austin, TX, 1997).

[7] F. Brezzi And M. Fortin, Mixed and hybrid finite element methods, Springer-Verlag, New York, 1991.

[8] A. N. Brooks And T. J. R. Hughes, Streamline upwind/Petrov-Galerkin formulations for convection dominated flows with particular emphasis on the incompressible Navier-Stokes equations, Comput. Methods Appl. Mech. Engrg., 32 (1982), pp. 199-259. FENOMECH '81, Part I (Stuttgart, 1981).

[9] P. G. Ciarlet, The finite element method for elliptic problems, vol. 40 of Classics in Applied Mathematics, Society for Industrial and Applied Mathematics (SIAM), Philadelphia, PA, 2002. Reprint of the 1978 original [North-Holland, Amsterdam; MR 58 \#25001]. 
[10] A. Cohen, W. Dahmen, And R. DeVore, Adaptive wavelet methods for elliptic operator equations: convergence rates, Math. Comp., 70 (2001), pp. 27-75 (electronic).

[11] A. Cohen, W. Dahmen, And R. DeVore, Adaptive wavelet methods. II. Beyond the elliptic case, Found. Comput. Math., 2 (2002), pp. 203-245.

[12] W. Dahmen, A. Kunoth, And R. Schneider, Wavelet least squares methods for boundary value problems, SIAM J. Numer. Anal., 39 (2002), pp. 1985-2013 (electronic).

[13] W. DÖRFLER, Uniform a priori estimates for singularly perturbed elliptic equations in multidimensions, SIAM J. Numer. Anal., 36 (1999), pp. 1878-1900 (electronic).

[14] H. Goering, A. Felgenhauer, G. Lube, H.-G. Roos, and L. Tobiska, Singularly perturbed differential equations, vol. 13 of Reihe Math. Research, Akademie-Verlag, Berlin, 1983.

[15] J.-L. Lions AND E. MAGENES, Non-homogeneous boundary value problems and applications. Vol. I, Springer-Verlag, New York, 1972. Translated from the French by P. Kenneth, Die Grundlehren der mathematischen Wissenschaften, Band 181.

[16] H.-G. Roos, M. Stynes, And L. Tobiska, Numerical methods for singularly perturbed differential equations, Springer-Verlag, Berlin, 1996. Convection-diffusion and flow problems.

[17] G. Sangalli, Analysis of the advection-diffusion operator using fractional order norms, Tech. Rep. 1221, I.A.N.-C.N.R., 2001. accepted on Numer. Math.

[18] _ Quasi-optimality of the SUPG method for the one-dimensional advection-diffusion problem, Tech. Rep. 1222, I.A.N.-C.N.R., 2001. accepted on SIAM J. Numer. Anal.

[19] H. TRIEBEL, Interpolation theory, function spaces, differential operators, Johann Ambrosius Barth, Heidelberg, second ed., 1995. 